\title{
Is there an association between long- term antibiotics for acne and subsequent infection sequelae and antimicrobial resistance? A systematic review
}

Ketaki Bhate, MBBS, MSc, MRCP (Dermatology) ${ }^{1 *}$, Liang-Yu Lin, MD, MSc ${ }^{1}$, John S Barbieri, MD², Clémence Leyrat, MSc, PhD ${ }^{1}$, Susan Hopkins, BA, MB BCh, BAO, MSc, FRCPI, FRCP ${ }^{3}$, Richard Stabler, $\mathrm{PhD}^{4}$, Laura Shallcross, BA, MBBS, MSc, PhD ${ }^{5}$, Liam Smeeth, MBChB, FRCGP, FFPH, FRCP, MSc, PhD, FMedSci ${ }^{1}$, Nick Francis, PhD, MD, BA, PGD (Epidemiology), MRCGP6, Rohini Mathur, BSc, MSc, PhD', Sinéad M Langan, FRCP, MSc, PhD ${ }^{1}$, Sarah-Jo Sinnott, BPharm, MPharm, PhD, MPSI ${ }^{1}$

${ }^{1}$ Faculty of Epidemiology and Population Health, London School of Hygiene and Tropical Medicine, London, UK; ${ }^{2}$ University of Pennsylvania, Perelman School of Medicine, Philadelphia, PA, USA; ${ }^{3}$ AMR Division, Public Health England, London, UK; ${ }^{4}$ Faculty of Infectious and Tropical Diseases, London School of Hygiene and Tropical Medicine, London, UK; ${ }^{5}$ Faculty of Population Health Sciences, University College London, London, UK; ${ }^{6}$ School of Primary Care, Population Sciences and Medical Education, University of Southampton, Southampton, UK

*For correspondence: ketaki. bhate@Ishtm.ac.uk

Competing interest: See page 8

Received: 07 December 2020 Accepted: 19 January 2021

Published: 07 April 2021

(C)This article is Open Access: CC BY license (https://creativecommons.org/licenses/by/4.0/)

Author Keywords: acne vulgaris, antibiotic, antimicrobial resistance, tetracycline, macrolides, dihydrofolate reductase inhibitor

Copyright (C) 2021, The Authors; DOI:10.3399/BJGPO.2020.0181

\section{Abstract}

Background: Antimicrobial resistance (AMR) is a global health priority. Acne vulgaris is a common skin condition for which antibiotic use ranges from a few months to years of daily exposure.

Aim: To systemically search for and synthesise evidence on the risk of treatment-resistant infections, and other evidence of AMR, following long-term oral antibiotic use for acne.

Design \& setting: In this systematic review, a literature search was carried out using the databases Embase, MEDLINE, Cochrane, and Web of Science. They were searched using MeSH, Emtree, or other relevant terms, and followed a pre-registered protocol.

Method: Search strategies were developed with a librarian and undertaken in July 2019. All searches date from database inception. The primary outcome was antibiotic treatment failure or infection caused by a resistant organism. Secondary outcomes included detection of resistant organisms without an infection, rate of infection, or changes to flora.

Results: A total of 6996 records were identified. Seventy-three full-text articles were shortlisted for full review, of which five were included. Two investigated rates of infection, and three resistance or changes to microbial flora. Three studies had 35 or fewer participants (range 20-118 496). Three studies had a serious or high risk of bias, one moderate, and one a low risk of bias. Weak evidence was found for an association between antibiotic use for acne and subsequent increased rates of upper respiratory tract infections and pharyngitis.

Conclusion: There is a lack of high quality evidence on the relationship between oral antibiotics for acne treatment and subsequent AMR sequelae. This needs to be urgently addressed with rigorously conducted studies. 


\section{How this fits in}

AMR is a global threat and the prolonged use of antibiotics in the treatment of skin conditions may contribute to this burden. Long-term oral antibiotics are frequently used to treat acne in relatively well, young adult populations. This review has highlighted the dearth of high quality studies on the implications of long-term oral antibiotic use on infectious or AMR sequelae. It is not understood how the long-term use of oral antibiotics for acne affects the subsequent rate of infections, changes to microbiota, or AMR. This systematic review has highlighted an urgent need for rigorous, wellconducted studies investigating the relationship between long-term antibiotics for acne and AMR.

\section{Introduction}

The World Health Organization has declared the threat of AMR a most urgent crisis. ${ }^{1}$ Currently, approximately 700000 people die per year as a result of AMR and a report predicted that there will be 50 million deaths per year as a result of AMR by 2050, with a total cumulative cost to lost global production of 100 trillion USD. ${ }^{2}$ Acne vulgaris is a chronic, inflammatory skin disorder, predominantly of adolescence. It affects $80-100 \%$ of adolescents, and $20 \%$ have moderate to severe acne. ${ }^{3}$ Topical and oral antibiotics are commonly prescribed in the treatment of acne. Although there is conflicting information in international acne guidelines, they generally recommend treatment with an oral or topical antibiotic for 3-6 months. ${ }^{4-9}$ Tetracyclines and macrolides are the two most common oral antibiotic classes prescribed for people with acne in UK primary care. ${ }^{4}$

The overuse of antibiotics is a cause of AMR. Exposures to antibiotics selects for bacteria with spontaneous or acquired mechanisms of resistance. In turn, commensal bacteria also develop and acquire mechanisms to resist the effects of antibiotics, which may give rise to invasive infection. While it is understood that acne is not an infectious disease and the pathophysiology of acne is multifactorial, with Cutibacterium acnes implied in one step in the development of an acne lesion, several studies have shown topical antibiotics for acne leads to resistant C. acnes. ${ }^{10-14}$ Less is known about whether antibiotic treatment for acne impacts on bacterial flora at other sites. Despite this, oral antibiotics are considered to have anti-inflammatory effects, and their short-term efficacy ensures continued use, alongside other treatments used for acne such as isotretinoin. ${ }^{15,16}$ Given the potential relationship between exposure to antibiotics and AMR, this practice may not be sustainable. ${ }^{17}$

Antimicrobial stewardship, a framework employed to ensure the judicious use of antibiotics, is effective for other infections in other settings; ${ }^{18}$ however, to ensure its implementation in acne treatment, evidence is needed to show that using antibiotics for acne increases future infective episodes and resistance sequelae. Until this evidence is obtained, there will be little impetus to change clinical practice. ${ }^{19}$

The question of whether antibiotics for acne contribute towards AMR is an evidence gap that needs to be urgently addressed. ${ }^{20}$ This study aims to address this gap by systematically reviewing published evidence on the association between long-term use of oral antibiotics for acne and subsequent risk of antibiotic treatment failure, infection caused by a resistant organism, or other evidence of AMR.

\section{Method}

The review protocol was registered on PROSPERO on 8 of April 2019 before the literature search ( www.crd.york.ac.uk/PROSPERO) and is published in BMJ Open. ${ }^{21}$ PRISMA (Preferred Reporting Items for Systematic Reviews and Meta-analyses) and RECORD (Reporting of studies Conducted Using Observational Routinely collected Data) guidance was followed. ${ }^{22}$

\section{Literature search strategy}

The databases Embase, MEDLINE, Cochrane, and Web of Science were searched. Search terms were developed by finding keywords from relevant articles and by running pilot searches. Searches were developed alongside a librarian to ensure completeness. To keep the searches as broad as possible the 'explode' function on Ovid was used. The search strategy was reviewed by all authors. The final searches were undertaken by the lead author who has medical and search training. Searches were undertaken in July 2019 from inception of the databases. 


\section{Inclusion and exclusion criteria}

The review included randomised controlled trials, and both cohort and case-control observational studies. Conference abstracts were included if the full article was unpublished but the full manuscript could be obtained from the authors. Studies were included if they met the above criteria in addition to the following criteria:

- The study population included participants aged $\geq 8$ years with acne, in any healthcare setting.

- The study investigated oral antibiotics prescribed for acne, for a minimum of 28 days of daily dosing.

- The comparison group included people who have not been treated with oral antibiotics for acne (or the general population).

- Studies where outcomes met the primary outcome of antibiotic treatment failure or infection caused by a resistant organism, or the secondary outcome of the detection of resistant organisms without an infection, rate of infection, or changes to bacterial flora. Any measure including proxy measures were used.

Ecological studies and studies that did not assess temporality or looked at specific subtypes of acne (for example, acne fulminans) were excluded. Unpublished, ongoing, and studies in grey literature were excluded. Studies that only looked at AMR of C. acnes or those including people aged $<8$ years were excluded, as acne vulgaris is unlikely to present in children aged $<8$ years and tetracyclines are not recommended in younger children.

\section{Exposure and comparator}

The exposure was at least 28 days of continuous daily doses of antibiotics for acne. This duration was chosen as 28 days is the usual minimum duration of therapy for acne and it was more likely to distinguish between people receiving antibiotics for acne and those receiving short-course antibiotics for an acute infection. Topical antibiotics were excluded as these are less likely to have an effect at sites other than the skin where they are applied. The comparator group included people with acne who were not treated with oral antibiotics or the general population.

\section{Outcome}

The primary outcome was antibiotic treatment failure (insufficient clinical improvement following treatment of an infection with an antibiotic), or any infection caused by a resistant organism. The secondary outcome was the detection of resistant organisms without a clinical infection, rate of infection, or changes to flora. This included: any measure of AMR, for example, laboratory measures (such as a raised C-reactive protein [an inflammatory marker, which if raised may support the diagnosis of a persistent infection despite prior treatment with an antibiotic or it can be used to monitor antibiotic treatment response to infection] or positive culture in the case of a subsequent resistant infection at any body site); patient observations (such as an elevated temperature and/or pulse rate [which may indicate an infection and could represent antibiotic treatment failure if persistent after treatment with an antibiotic]); or proxy measures that may have been used in epidemiological studies, for example, difficult-to-treat infections. Antibiotic treatment failure is a proxy for AMR. The outcome could occur at any time point after at least 28 days of continuous oral antibiotic exposure for acne. Outcome measures were developed a priori.

\section{Eligibility assessment and data extraction}

Covidence, an online literature review data management programme, was used to facilitate the systematic review process. ${ }^{23}$ All titles and abstracts were uploaded to Covidence. Duplicates were removed and three reviewers - KB, LYL, and JB — independently screened the search results based on title and abstract. Each title and/or abstract needed two votes to undergo full-text review. Conflicts were resolved by the involvement of a fourth reviewer not involved in the screening process, SML.

Full-text articles were assessed independently by the same reviewers. The extraction of the first included record was piloted by all reviewers and discrepancies were discussed. The Cochrane Risk of Bias 2 (RoB 2) tool was used to assess the risk of bias in randomised studies and Risk of Bias in Non-randomised Studies - of Interventions (ROBINS-I) tool was used to assess the risk of bias in nonrandomised studies. ${ }^{24,25}$ Grading of Recommendations, Assessment, Development and Evaluations 


\section{Records identified through database searching $(n=8492)$ \\ Medline: $n=3170$ \\ Embase: $\mathrm{n}=\mathbf{2 6 1 6}$ \\ Web of Science: $\mathbf{n = 2 7 0 6}$ \\ Cochrane: $n=0$}

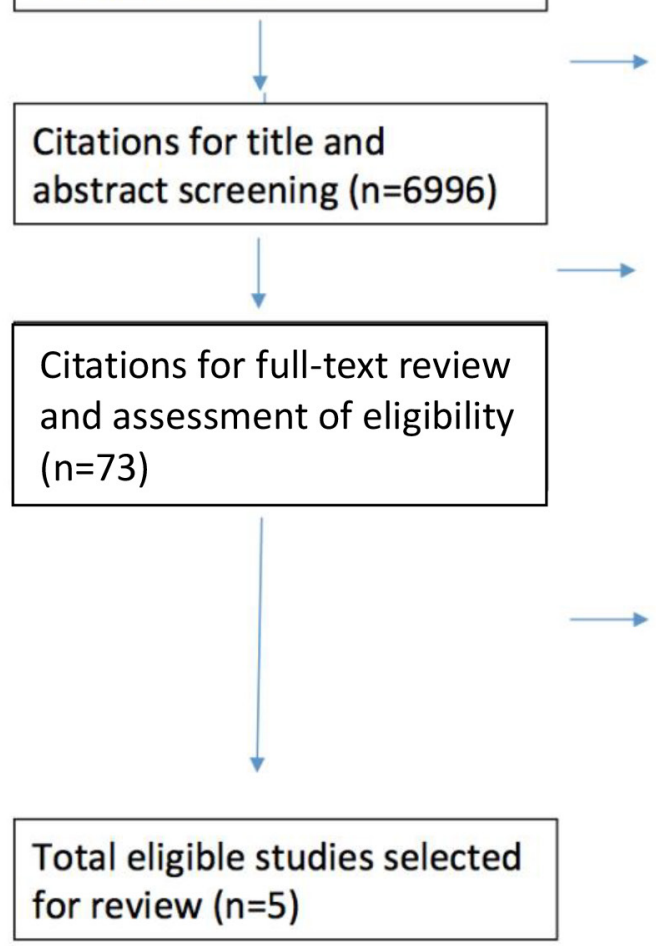

Duplicates records removed $(n=1496)$

Conflicts resolved $\mathrm{n}=104$

Records excluded: $n=6923$

Figure 1 Flow diagram of study selection

(GRADE) was used to make an overall assessment of the quality of evidence. ${ }^{26}$ Pairs of reviewers made independent assessments of the risk of bias.

\section{Results}

A total of 6996 records were identified for title and abstract screening after de-duplication (Figure 1). Of these, 73 full-text articles were shortlisted for full-text review. The full-text of one study could not be obtained despite contacting library repositories in both the UK and US as well as contacting authors; this study was therefore excluded. Overall, five studies were included in the systematic review. ${ }^{27-31}$ The reasons the full-text articles were excluded are in Supplemetary Appendix A. The characteristics of the included studies are summarised in supplementary Table 1, and study results, risk of bias, and overall GRADE assessment are summarised in supplementary Table 2 and Tables 1-3.

\section{Study characteristics}

None of the five included studies measured the primary outcomes; three studies investigated the carriage or AMR bacteria using bacterial culture methods, and two studies investigated the rate of infection following antibiotics for acne. Only one study was a randomised controlled trial; ${ }^{30}$ the remaining four were all cohort studies, two of which were undertaken involving patients solely in the UK, and one of those used routinely collected medical records from UK general practice. All studies were from high or upper-middle income countries (three studies from the UK, one from Sweden, and 


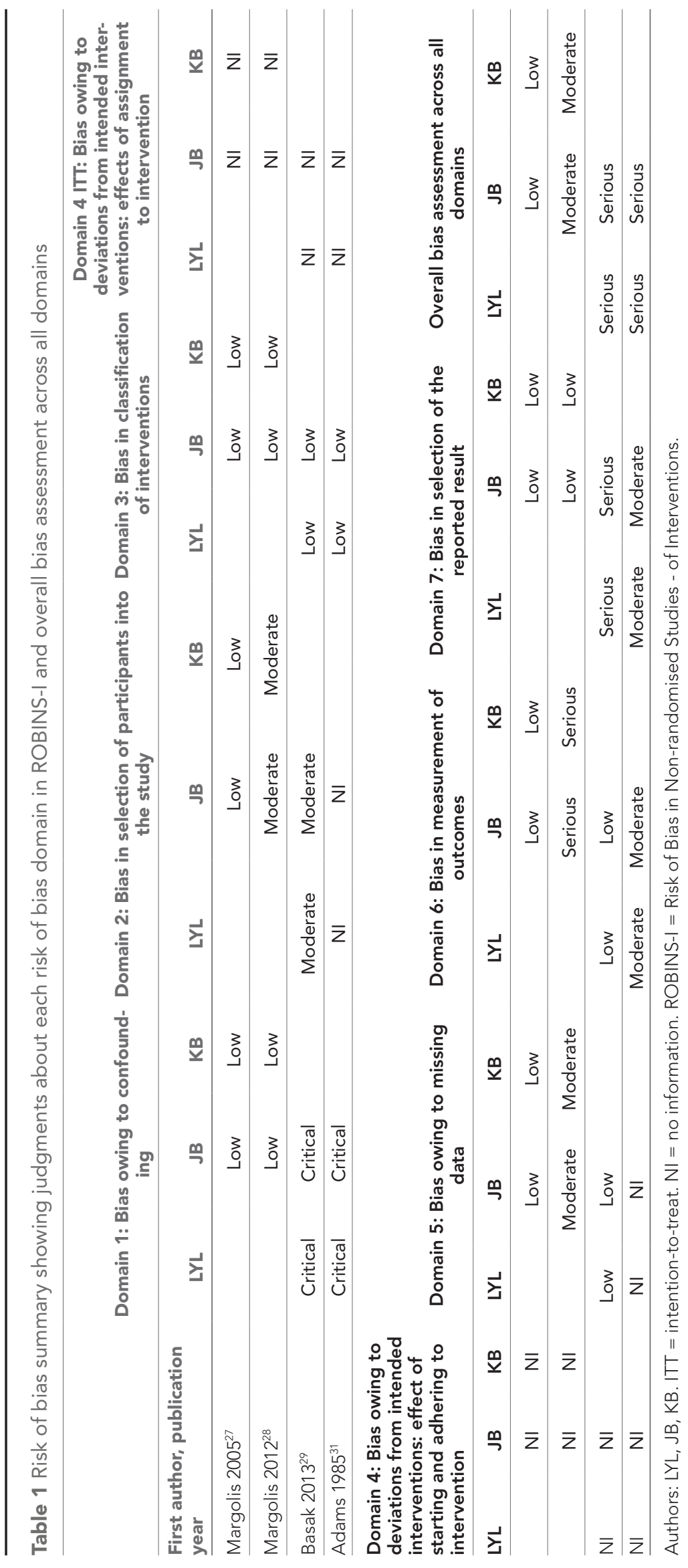


Table 2 The Risk of Bias 2 (RoB 2) assessment for randomised controlled trial ${ }^{30}$

\begin{tabular}{lcc}
\hline Borglund et $^{\mathbf{B}^{30}}$ RoB 2 & LYL & KB \\
\hline Domain 1 Randomisation process & High & High \\
\hline $\begin{array}{l}\text { Domain 2 Deviations from intended } \\
\text { interventions }\end{array}$ & High & High \\
\hline Domain 3 Missing outcome data & Low & Low \\
\hline Domain 4 Measurement of the outcome & Some concerns & Some concerns \\
\hline Domain 5 Selection of the reported results & Some concerns & Some concerns \\
\hline Overall risk of bias & High & High \\
\hline
\end{tabular}

Authors: LYL, KB.

one from Turkey). Study sizes ranged from 20-118 496 participants, and three studies had 35 or fewer included individuals. The mean age of study participants ranged from 17.6-21.7 years (age range 15-38 years).

Given the heterogeneity of included studies, particularly with regard to outcomes, it was not possible to perform a meta-analysis. Therefore, the results of this systematic review are reported narratively.

Borglund et $a^{30}$ investigated changes in the quantity and resistance patterns of skin and intestinal flora in a randomised controlled trial comparing topical clindamycin 1\% along with a tablet placebo, and tetracycline $250 \mathrm{mg}$ twice a day orally along with a topical placebo. ${ }^{30}$ The authors reported pronounced reductions in the numbers of streptococci, enterococci, fusobacteria, and enterobacteria in the colon during the treatment period with oral tetracycline and, in particular, new colonisation with tetracycline-resistant strains was noted. The flora normalised to pre-treatment levels 8 weeks

Table 3 Summary of findings (GRADE assessment of quality of evidence)

\begin{tabular}{|c|c|c|c|c|c|c|c|c|}
\hline \multicolumn{9}{|c|}{ Summary of findings } \\
\hline $\begin{array}{l}\text { Number of } \\
\text { studies }\end{array}$ & Study design & Risk of bias & Inconsistency & Indirectness & Imprecision & $\begin{array}{l}\text { Other consider- } \\
\text { ations }\end{array}$ & $\begin{array}{l}\text { Number of } \\
\text { patients }\end{array}$ & Quality \\
\hline \multicolumn{9}{|c|}{ Rate of infection } \\
\hline 2 & Cohort & Not serious & Not serious & Not Serious & Serious & $\begin{array}{l}\text { No: publication } \\
\text { bias, large } \\
\text { effect, plausible } \\
\text { confounding, } \\
\text { dose response } \\
\text { gradient }\end{array}$ & $\begin{array}{l}\text { Intervention } \\
\text { total: } 79807, \\
\text { Control total: } \\
33792\end{array}$ & $\begin{array}{l}\oplus \bigoplus \\
\mathrm{LOW} a, b\end{array}$ \\
\hline
\end{tabular}

Detection of resistant organisms without an infection or changes to flora or microbiota

\begin{tabular}{|c|c|c|c|c|c|c|c|c|}
\hline 3 & $\begin{array}{l}1 \mathrm{RCT} \text { and } 2 \\
\text { cohort studies }\end{array}$ & Serious & Not serious & Not serious & Serious & $\begin{array}{l}\text { No: publication } \\
\text { bias, large } \\
\text { effect, plausible } \\
\text { confounding, } \\
\text { dose response } \\
\text { gradient }\end{array}$ & $\begin{array}{l}\text { Intervention } \\
\text { total: } 36 \text {, Control } \\
\text { total: } 45\end{array}$ & $\begin{array}{l}\oplus \\
\text { VERY LOW } \\
c, d, e, f, d, g\end{array}$ \\
\hline
\end{tabular}

Explanations

a. Selection bias: students selected from one university campus.

b. Imprecise estimates: wide $95 \%$ confidence intervals.

c. Selection bias: patients not randomised to treatment.

d. Confounding factors not reported or incorporated in analysis.

e. Follow-up inconsistent between treatment groups.

f. Confidence intervals not reported and small sample size.

g. No 95\% confidence intervals reported: predominantly numbers and percentages reported.

GRADE = Grading of Recommendations Assessment, Development and Evaluation; RCT = randomised controlled trial. 
after treatment was stopped. Resistance to tetracycline during treatment was seen in $40 \%$ of the staphylococcal and enterococcal isolates from the skin.

Two of the studies Margolis et al $2005^{27}$ and Margolis et al $2012^{28}$ investigated the rate of infections following the use of antibiotics for acne. The first used routinely collected electronic health records from the UK (Clinical Practice Research Datalink, formerly General Practice Research Datalink) ( $n=118$ 496) to evaluate the association between oral antibiotics prescribed for acne and subsequent upper respiratory tract infections (URTI) and urinary tract infections (UTI). ${ }^{27}$ The authors identified statistically significant associations between being prescribed a long-term oral antibiotic for acne $(n=197)$ and having a subsequent consultation coded for a URTI (odds ratio [OR] $=2.75$ (95\% confidence interval $[\mathrm{Cl}]=2.37$ to 3.18 ) or $\mathrm{UTI}$ (in women; $\mathrm{OR}=1.87$ [95\% $\mathrm{Cl}=1.38$ to 2.53$]$; information received via communication with authors [numbers of UTI in men too small for analysis]). The number of individuals with a UTI diagnosis who had received an oral antibiotic for their acne was not reported.

The second study by Margolis et al was a cohort study in 2012 ( $n=579$ ), which investigated the risk of developing pharyngitis in students with acne receiving antibiotic treatment who were based on one university campus in North America. ${ }^{28}$ Thirty-six (6.2\%) individuals took an oral antibiotic for their acne. Four out of $36(11.3 \%)$ of those taking an antibiotic for acne reported an episode of pharyngitis compared with 18 out of 543 (3.3\%) of those not taking an antibiotic for their acne. The OR associating oral antibiotic use with pharyngitis was $4.34(95 \% \mathrm{Cl}=1.51$ to 12.47$)$ using mixed model multivariable regression.

The final two studies investigated changing resistance patterns among flora following exposure to oral antibiotics for acne. Adams et al studied the changing pattern of bowel flora resistance in 26 individuals comprising patients with acne receiving oral erythromycin $(n=6)$ and tetracycline $(n=5)$ and family members living in the same household as the patient with acne. ${ }^{31}$ Patients who had received tetracycline for acne and their relatives developed greater numbers of tetracycline Escherichia coli resistant isolates. Conversely, the numbers of erythromycin-resistant $E$. coli isolates decreased in acne patients receiving an antibiotic for acne but increased in their relatives.

The other study aimed to investigate changes in the microbial flora of the nose, oropharynx, and faeces following use of systemic isotretinoin $(n=20)$ and oral antibiotic therapy $(n=15) \cdot{ }^{29}$ The authors described it as a randomised controlled trial, however, patients were placed into treatment groups based on acne severity with no description of any random element to treatment allocation. The methods stated that logistic regression was used in analyses, however, no odds ratios were presented. The study reported that antibiotics caused less differentiation (which authors defined as the isolation of Salmonella spp., Shigella spp., Pseudomonas aeruingosa and extended-spectrum beta-lactamase [ESBL] gram negative bacilli) of microbial flora compared with isotretinoin at all the cultured sites.

\section{Discussion}

\section{Summary}

This systematic review found five studies that met the inclusion criteria. All studies investigated secondary outcomes: the detection of resistant organisms without an infection or the rate of infection. No studies in the review addressed the primary outcome of antibiotic treatment failure or infection caused by a resistant organism. Overall, across all outcomes, low or very low quality of evidence was found supporting long-term oral antibiotics for acne being associated with infectious outcomes or AMR (Table 3).

The mechanisms for how $C$. acnes (the bacterium pathophysiologically implicated in the formation of an acne lesion) becomes resistant to topical antibiotics used to treat acne are well described, but oral antibiotic treatments for acne are distributed throughout the body, and the impact of their use on the spread of AMR and risk of treatment-resistant infections is not fully understood. ${ }^{32,33}$ There are reviews aiming to summarise the evidence of AMR secondary to antibiotics for acne; however, this is the first systematic review to the authors' knowledge that aims to address infectious outcomes and resistance of flora other than $C$. acnes as a result of oral antibiotics for acne.

\section{Strengths and limitations}

Strengths of the systematic review included: following a pre-specified protocol published on PROSPERO and BMJ Open; designing and reporting the review following PRISMA guidance; 
undertaking a comprehensive search developed in collaboration with a librarian; having no language or time limits; and completing a full bias risk assessment and reporting the overall quality of evidence using GRADE. In addition, the screening process was undertaken by medical healthcare professionals with epidemiological training. ${ }^{22}$ Limitations included not searching the grey literature, and the lack of studies from developing countries where antibiotics may be used for acne and may be bought over the counter.

\section{Implications for research and practice}

This review has highlighted the dearth of high quality scientific research on the implications of longterm oral antibiotic use for acne on infectious or AMR sequelae. The impact that use of oral antibiotics for acne has on microbial resistance in commensal organisms and difficult-to-treat infections caused by organisms resistant to common antibiotics remains unclear. The degree to which cross-resistance to antibiotic classes other than the one prescribed for acne is also unclear. ${ }^{34,35}$ Given the predicted impact of AMR on death rates — in the order of one death every 3 seconds by 2050 - and the widespread use of long-term oral antibiotics for acne in a relatively healthy, young population, ${ }^{2}$ it is imperative to understand how these antibiotics may contribute to the burden of AMR with high quality prospective studies, so that practice can be modified if needed.

\section{Funding}

KB is funded by an NIHR Doctoral Research Fellowship DRF-2018-11-ST2-066. SL is funded by a Wellcome Trust Senior Clinical Fellowship (205039/Z/16/Z). JB is supported by the National Institute of Arthritis and Musculoskeletal and Skin Disease of the National Institutes of Health under award number T32-AR-007465. CL is funded by an MRC Skills Development Fellowship (MR/T032448/1). LS is funded by an NIHR Clinician Scientist Award (CS-2016-16-007). RM is funded by a Wellcome Trust Postdoctoral Fellowship (201375/Z/16/Z). The views expressed are those of the author(s) and not necessarily those of the NHS, the NIHR or the Department of Health and Social Care.

Ethical approval

No ethical approval was required for this systematic review.

\section{Registration number}

The review protocol was registered on PROSPERO on 8 April 2019 prior to literature search, (CRD42019121738 - www.crd.york.ac.uk/PROSPERO)

\section{Provenance}

Freely submitted; externally peer reviewed.

\section{Competing interests}

John Barbieri receives partial salary support through a Pfizer Fellowship grant to the Trustees of the University of Pennsylvania.

\section{References}

1. World Health Organization. Global action plan on antimicrobial resistance. 2015; https://www.who.int/ antimicrobial-resistance/publications/global-action-plan/en/ (accessed 18 Mar 2021).

2. O'Neill J. Tackling drug-resistant infections globally: final report and recommendations. The review on antimicrobial resistance. 2016; https://amr-review.org/sites/default/files/160518_Final\%20paper_with\%20cover.pdf (accessed 18 Mar 2021).

3. Bhate K, Williams HC. Epidemiology of acne vulgaris. Br J Dermatol 2013; 168(3): 474-485. DOI: https://doi.org/ 10.1111/bjd.12149

4. Barbieri JS, Hoffstad O, Margolis DJ. Duration of oral tetracycline-class antibiotic therapy and use of topical retinoids for the treatment of acne among general practitioners (GP): a retrospective cohort study. J Am Acad Dermatol 2016; 75(6): 1142-1150. DOI: https://doi.org/10.1016/j.jaad.2016.06.057

5. Lee $\mathrm{YH}$, Liu G, Thiboutot DM, et al. A retrospective analysis of the duration of oral antibiotic therapy for the treatment of acne among adolescents: investigating practice gaps and potential cost-savings. J Am Acad Dermatol 2014; 71(1): 70-76. DOI: https://doi.org/10.1016/j.jaad.2014.02.031

6 Whitehouse HJ FE, El-Mansori I, Layton AM. [Conference presentation] Oral antibiotics for acne: are we adopting premium use? British Association of Dermatologists Annual Conference; Birmingham. 5-7 Jul 2016. 
7. National Institute for Health and Care Excellence. Clinical knowledge summaries. Acne vulgaris. London: NICE; 2014.

8. Zaenglein $A L$, Pathy $A L$, Schlosser $B J$, et al. Guidelines of care for the management of acne vulgaris. J Am Acad Dermatol 2016; 74(5): 945-973. DOI: https://doi.org/10.1016/j.jaad.2015.12.037

9. Nast A, Dréno B, Bettoli V, et al. European evidence-based (S3) guideline for the treatment of acne — update 2016 — short version. J Eur Acad Dermatol Venereol 2016; 30(8): 1261-1268. DOI: https://doi.org/10.1111/jdv.13776

10. Kuet KH, FE FC, Eady A, Layton A. [Conference presentation] A decade later, has the prevalence of skin colonization by resistant propionibacteria increased in our patients with acne? British Association of Dermatologists Annual Conference; Manchester. 7-9 Jul 2015.

11. Lee SE, Kim J-M, Jeong SK, et al. Protease-activated receptor-2 mediates the expression of inflammatory cytokines, antimicrobial peptides, and matrix metalloproteinases in keratinocytes in response to Propionibacterium acnes. Arch Dermatol Res 2010; 302(10): 745-756. DOI: https://doi.org/10.1007/s00403-010-1074-z

12. Ross Jl, Snelling AM, Carnegie E, et al. Antibiotic-resistant acne: lessons from Europe. Br J Dermatol 2003; 148(3): 467-478. DOI: https://doi.org/10.1046/j.1365-2133.2003.05067.x

13. Leyden JJ, McGinley KJ, Cavalieri S, et al. Propionibacterium acnes resistance to antibiotics in acne patients. $J$ Am Acad Dermatol 1983; 8(1): 41-45. DOI: https://doi.org/10.1016/S0190-9622(83)70005-8

14. Crawford WW, Crawford IP, Stoughton RB, Cornell RC. Laboratory induction and clinical occurrence of combined clindamycin and erythromycin resistance in Corynebacterium acnes. J Invest Dermatol 1979; 72(4): 187-190. DOI: https://doi.org/10.1111/1523-1747.ep12676385

15. Ozolins M, Eady EA, Avery AJ, et al. Comparison of five antimicrobial regimens for treatment of mild to moderate inflammatory facial acne vulgaris in the community: randomised controlled trial. Lancet 2004; 364(9452): 21882195. DOI: https://doi.org/10.1016/S0140-6736(04)17591-0

16. Vallerand IA, Lewinson RT, Farris MS, et al. Efficacy and adverse events of oral isotretinoin for acne: a systematic review. Br J Dermatol 2018; 178(1): 76-85. DOI: https://doi.org/10.1111/bjd.15668

17. Bienenfeld A, Nagler AR, Orlow SJ. Oral antibacterial therapy for acne vulgaris: an evidence-based review. Am J Clin Dermatol 2017; 18(4): 469-490. DOI: https://doi.org/10.1007/s40257-017-0267-z

18. Lawes T, Lopez-Lozano J-M, Nebot CA, et al. Effects of national antibiotic stewardship and infection control strategies on hospital-associated and community-associated meticillin-resistant Staphylococcus aureus infections across a region of Scotland: a non-linear time-series study. Lancet Infect Dis 2015; 15(12): 1438-1449. DOI: https:// doi.org/10.1016/S1473-3099(15)00315-1

19. Simpson SA, Wood F, Butler CC. General practitioners' perceptions of antimicrobial resistance: a qualitative study. J Antimicrob Chemother 2007; 59(2): 292-296. DOI: https://doi.org/10.1093/jac/dkl467

20. Sinnott S-J, Bhate K, Margolis DJ, Langan SM. Antibiotics and acne: an emerging iceberg of antibiotic resistance? Br J Dermatol 2016; 175(6): 1127-1128. DOI: https://doi.org/10.1111/bjd.15129

21. Bhate K, Lin L-Y, Barbieri J, et al. Is there an association between long-term antibiotics for acne and subsequent infection sequelae and antimicrobial resistance? A systematic review protocol. BMJ Open 2020; 10(7): e033662. DOI: https://doi.org/10.1136/bmjopen-2019-033662

22. Shamseer L, Moher D, Clarke M, et al. Preferred reporting items for systematic review and meta-analysis protocols (PRISMA-P) 2015: elaboration and explanation. BMJ 2015; 350: g7647: g7647: . DOI: https://doi.org/10.1136/bmj. g7647

23. Covidence. Better systematic review management; www.covidence.org (accessed 18 Mar 2021)

24. Sterne JA, Hernán MA, Reeves BC, et al. ROBINS-I: a tool for assessing risk of bias in non-randomised studies of interventions. BMJ 2016; 355: i4919. DOI: https://doi.org/10.1136/bmj.i4919

25. Higgins JPT, Altman DG, Gøtzsche PC, et al. The Cochrane Collaboration's tool for assessing risk of bias in randomised trials. BMJ 2011; 343: d5928: d5928: . DOI: https://doi.org/10.1136/bmj.d5928

26. Schünemann H, Brożek J, Guyatt G. GRADE Handbook. Handbook for grading the quality of evidence and the strength of recommendations using the GRADE approach (updated October 2013). 2013; https://gdt.gradepro. org/app/handbook/handbook.html (accessed 18 Mar 2021).

27. Margolis DJ, Bowe WP, Hoffstad O, Berlin JA. Antibiotic treatment of acne may be associated with upper respiratory tract infections. Arch Dermatol 2005; 141(9): 1132-1136. DOI: https://doi.org/10.1001/archderm.141.9. 1132

28. Margolis DJ, Fanelli M, Kupperman E, et al. Association of pharyngitis with oral antibiotic use for the treatment of acne: a cross-sectional and prospective cohort study. Arch Dermatol 2012; 148(3): 326-332. DOI: https://doi.org/ 10.1001/archdermatol.2011.355

29. Başak PY, Cetin ES, Gürses I, Ozseven AG. The effects of systemic isotretinoin and antibiotic therapy on the microbial floras in patients with acne vulgaris. J Eur Acad Dermatol Venereol 2013; 27(3): 332-336. DOI: https:// doi.org/10.1111/j.1468-3083.2011.04397.x

30. Borglund E, Hägermark O, Nord CE. Impact of topical clindamycin and systemic tetracycline on the skin and colon microflora in patients with acne vulgaris. Scand J Infect Dis Suppl 1984; 43: 76-81.

31. Adams SJ, Cunliffe WJ, Cooke EM. Long-Term antibiotic therapy for acne vulgaris: effects on the bowel flora of patients and their relatives. J Invest Dermatol 1985; 85(1): 35-37. DOI: https://doi.org/10.1111/1523-1747. ep12275004

32. Hartley $\mathrm{CL}$, Richmond $\mathrm{MH}$. Antibiotic resistance and survival of $E$ coli in the alimentary tract. $\mathrm{Br} \mathrm{Med} J \mathrm{~J}$ (975; 4(5988): 71-74. DOI: https://doi.org/10.1136/bmj.4.5988.71

33. Valtonen MV, Valtonen VV, Salo OP, Mäkelä PH. The effect of long term tetracycline treatment for acne vulgaris on the occurrence of $\mathrm{R}$ factors in the intestinal flora of man. Br J Dermatol 1976; 95(3): 311-316. DOI: https://doi.org/ 10.1111/j.1365-2133.1976.tb07019.x 
34. Espersen F. Resistance to antibiotics used in dermatological practice. Br J Dermatol 1998; 139 Suppl 53: 4-8. DOI: https://doi.org/10.1046/j.1365-2133.1998.1390s3004.x

35. Vowels BR, Feingold DS, Sloughfy C, et al. Effects of topical erythromycin on ecology of aerobic cutaneous bacterial flora. Antimicrob Agents Chemother 1996; 40(11): 2598-2604. DOI: https://doi.org/10.1128/AAC.40.11. 2598 